Cite this: Phys. Chem. Chem. Phys., 2011, 13, 21063-21069

\title{
Rapid probe of the nicotine spectra by high-resolution rotational spectroscopy $\dagger$
}

\author{
Jens-Uwe Grabow, ${ }^{a}$ S. Mata, ${ }^{b}$ José L. Alonso, ${ }^{* b}$ I. Peña, ${ }^{b}$ S. Blanco, ${ }^{b}$ \\ Juan C. López ${ }^{b}$ and C. Cabezas ${ }^{b}$ \\ Received 4th July 2011, Accepted 30th September 2011 \\ DOI: $10.1039 / \mathrm{c} 1 \mathrm{cp22197c}$
}

Nicotine has been investigated in the gas phase and two conformational forms were characterized through their rotational spectra. Two spectroscopic techniques have been used to obtain the spectra: a new design of broadband Fourier transform microwave (FTMW) spectroscopy with an in-phase/quadrature-phase-modulation passage-acquired-coherence technique (IMPACT) and narrowband FTMW spectroscopy with coaxially oriented beam-resonator arrangement (COBRA). The rotational, centrifugal distortion and hyperfine quadrupole coupling constants of two conformers of nicotine have been determined and found to be in $N$-methyl trans configurations with the pyridine and pyrrolidine rings perpendicular to one another. The quadrupole hyperfine structure originated by two ${ }^{14} \mathrm{~N}$ nuclei has been completely resolved for both conformers and used for their unambiguous identification.

\section{Introduction}

Nicotine is an alkaloid abundantly present in the leaves of the tobacco plant and for that reason it is principally related with cigars and cigarettes and thus of main importance in the corresponding nervous and health disorders. ${ }^{1}$ Nevertheless, this compound has a wide range of uses in therapeutics, ${ }^{1}$ insecticides $^{2}$ and formation of polymers. ${ }^{3}$ The biological activity of nicotine, an agonist of the nicotine acetylcholine receptor protein, depends on its molecular shape, since active sites in receptors and enzymes would interact only with molecules of the correct conformation. Thus the knowledge of the three dimensional arrangements of various conformers plays a crucial role.

Experimental data on nicotine are available in condensed phases. Neat and hydrated protonated nicotine ions have been the subject of a rather large number of experimental investigations. ${ }^{4-12}$ However, experiments in condensed phases, where package or solvent effects are present, do not lead to the intrinsic conformational preferences of nicotine. These can only be obtained in the gas phase, where inherent properties can be

${ }^{a}$ Institut für Physikalische Chemie und Elektrochemie, Lehrgebiet A, Gottfried-Wilhelm-Leibniz-Universität Hannover, Callinstraße 3 A, D-30167 Hannover, Germany

${ }^{b}$ Grupo de Espectroscopia Molecular (GEM), Edificio Quifima,

Laboratorios de Espectroscopia y Bioespectroscopia, Parque

Cientifico Uva, Universidad de Valladolid, 47005 Valladolid, Spain.

E-mail: jlalonso@qf.uva.es; Fax: +34 983186349,

Tel: +34983186348

$\dagger$ Electronic supplementary information (ESI) available: Complete list of transition frequencies measured for the two nicotine conformers and complete ref. 35. See DOI: $10.1039 / \mathrm{clcp} 22197 \mathrm{c}$ discriminated from external bias, since the interaction of an isolated molecule with the environment can be precisely controlled. The first structural study concerning neutral nicotine has been conducted by gas-phase electron diffraction in combination with theoretical calculations. ${ }^{13}$ The electron diffraction pattern was reproduced by assuming a mixture of two distinct conformers of nicotine. Studies of the nicotine analogues 2-phenylpyrrolidine ${ }^{14}$ and anabasine ${ }^{15}$ have been recently reported. The former has been studied in gas and solution-phase using MW, electronic and NMR spectroscopic techniques. ${ }^{14}$ Although this molecule retains the key two-ring structural motif of nicotine, the replacement of the pyridine ring by a phenyl group removes the hydrogen bond acceptor site at the pyridine nitrogen. In anabasine, where the pyrrolidine moiety is substituted by a piperidine ring, two conformers have been observed by rotational spectroscopy. ${ }^{15}$

The present study on nicotine follows our interest to examine relevant biomolecules by the high resolution of microwave spectroscopic techniques. The combination of supersonic-jets with Fourier transform microwave (FTMW) spectroscopy ${ }^{16-18}$ has proved to be a powerful tool in the investigation of the conformational behavior of gas phase biomolecular systems. In such studies, vaporization of biomolecules is achieved through the laser ablation molecular beam (LA-MB-FTMW) technique $^{19,20}$ or heating methods. ${ }^{21,22}$ All the conformational species with significant population in the adiabatic expansion can be independently studied through their rotational spectrum, just as in the case of a mixture of stable nonreactive species.

The first investigation on the rotational spectra of nicotine was done by Lavrich et al. and the results were presented at the 58 th International Symposium on Molecular Spectroscopy. ${ }^{23}$ 
In that work the authors provided the rotational constants of two conformers obtained from a fit of the estimated line centers. In the present work, which was also previously presented as a communication to the same conference in its 65 th edition, ${ }^{24}$ we have analyzed the rotational spectra of nicotine using a new design of a linear fast passage (chirp) FTMW spectrometer ${ }^{25-27}$ called IMPACT complemented with the established setup of a short stimulus (pulse) excitation FTMW spectrometer of the COBRA type. This new approach provides instant broadband performance using fast linear frequency sweeps (chirps), instead of on/off-modulated CW radiation (pulses), to polarize the sample. Details of the design are given in the next section. Narrow splittings due to nuclear quadrupole coupling interactions were resolved and analyzed. Assignments of the observed spectra to different conformers were made with the aid of high level ab initio calculations based on the comparison of observed and calculated spectroscopic constants. The analysis leads to the unequivocal identification of the two low-energy conformers of nicotine.

\section{Experimental details}

The spectra were obtained using the two different FTMW spectrometers constructed at the University of Valladolid. A commercial sample of nicotine was used directly, without any further purification. For the experiments described here, a sample of liquid nicotine was placed in a reservoir close to the nozzle and heated to $60{ }^{\circ} \mathrm{C}$. For the IMPACT-FTMW setup two reservoirs were used, each coupled to one pulsed valve to seed nicotine in the carrier gas, $\mathrm{Ne}$, at a backing pressure of 2 bar, preceding the supersonic expansion.

\section{(a) IMPACT-FTMW}

A block diagram of the supersonic-jet expansion linear fast passage (FP) FTMW spectrometer discussed here is presented in Scheme 1. While the general design considerations regarding its high-frequency electronics are instructive for FP-based methods in general, ${ }^{28}$ it provides performance advantages in some specific points. ${ }^{29}$ The design implements the in-phase/ quadrature-phase-modulation passage-acquired-coherence technique (IMPACT) by using a single-stage in-phase/quadraturephase (I/Q) modulation scheme for direct single-sideband frequency conversion. The IMPACT spectrometer (see Scheme 1) uses a short MW radiation frequency ramp for excitation and in this particular implementation uses components that allow for frequency coverage from $8-18 \mathrm{GHz}$ (with appropriate antennae). The principle of operation of this relatively simple set-up - compared to typical FTMW instruments-is as follows: a primary signal with frequency $\nu$ is generated by the signal generator (1). Employing a pin-diode singlepole-double-throw (SPDT) switch (2), this signal is either used for excitation or for detection. In the first case, a short MW frequency ramp centered at $\nu$ has to be created with enough microwave power for the FP excitation of the molecules. In the second case, the microwave response of the probe has to be converted - taken as a difference frequency signal between the molecular and the primary radiation at frequency $\nu$-into a lower-frequency band, the so-called base band. Similar to the coaxially oriented beam-resonator arrangement (COBRA)
FT-MW apparatus, ${ }^{30-32}$ the primary signal source is not used directly for the molecular excitation. The output signal of a single-sideband up-converter (4) is utilized instead. However, in contrast to the narrow-band $(<1 \mathrm{MHz})$ excitation scheme of the COBRA apparatus, where the single-sideband modulator is used to generate a specific fixed frequency, in the IMPACT instrument a broad-band $(<1 \mathrm{GHz})$ single-sideband frequency ramp $\nu+\Delta \nu(t)$ is created via $\mathrm{I} / \mathrm{Q}$ modulation at frequencies $\Delta \nu(t)$ in the range -500 to $+500 \mathrm{MHz}$, centered at dc, invoking an arbitrary-signal generator (3). As a consequence of two RF signals swept from $500 \mathrm{MHz}$ through dc and back to $500 \mathrm{MHz}$ while alternating the quadrature phase sense, two low and high frequency half-bands are generated, thus creating the broad-band frequency ramp centered at $\nu$. This ramp, with its generation principle inherently warranting for invariant phase repeatability, is used for excitation. The power level necessary for the polarization of the molecular systems is adjusted either via the amplitude of the modulation frequency or using a variable attenuator (5). A combination of a fixed attenuator (6) and a medium power amplifier (7) sets a safe maximum input level for the high-power travelling-wave-tube (TWT) amplifier (8). High power levels become necessary because of the spectral width of $1 \mathrm{GHz}$, which is swept in $\tau_{\mathrm{p}}=10 \mu$ s typically.

The pin-diode single-pole-single-throw (SPST) switch (11) isolates the molecular interaction zone during the detection from noise of the excitation electronics. The idle noise between the excitation pulses is reflected by the pin-diode SPST switch and eliminated in the isolator (9). After passing the pin-diode switch, the energy of the excitation signal emerges from the horn antenna (13a) eventually interfering with the region of the jet-expansion originating from the solenoid valves (22) and thus providing the MW field amplitude for molecular polarization. The molecular IMPACT response, containing signals within the range $\nu-500$ to $\nu+500 \mathrm{MHz}$ is then received by the opposite horn antenna (13b) and passes the power limiter (14) which protects the delicate MW receiver electronics from the harmful power levels of the frequency ramp excitation.

The molecular signal is passed to a very-low-noise amplifier (15) followed by a second low-noise amplifier (16). The combined gain is chosen to be just sufficiently high for the insertion losses in the subsequent stages of the signal processing only having a negligible impact on the $\mathrm{S} / \mathrm{N}$ ratio, thus realizing the widest dynamic range at optimum sensitivity. In the singlesideband modulator (17) the broad-band molecular IMPACT response is converted back into the base band centered at dc, with $\Delta \nu=-500$ to $+500 \mathrm{MHz}$ created as $\mathrm{I} / \mathrm{Q}$ signals, i.e. negative and positive frequency signals differing in quadrature phase sense. By selecting the appropriate primary frequency $\nu$, any desired $1 \mathrm{GHz}$ band region of the spectrum within the range 2-26.5 GHz can be projected into this base band. The frequency conversion from and back to the base band in a single step minimizes jitter, i.e. destructive phase fluctuations. The lowpass filters (18) reject signals beyond the Nyquist frequency limit according to the folding theorem of the discrete FT. The A/D-conversion is accomplished by the transient recorder (19), which provides two-channel quadrature detection. After performing a complex FT in the data acquisition, control, and analysis system (20), the spectral composition of the transient emission is obtained. 


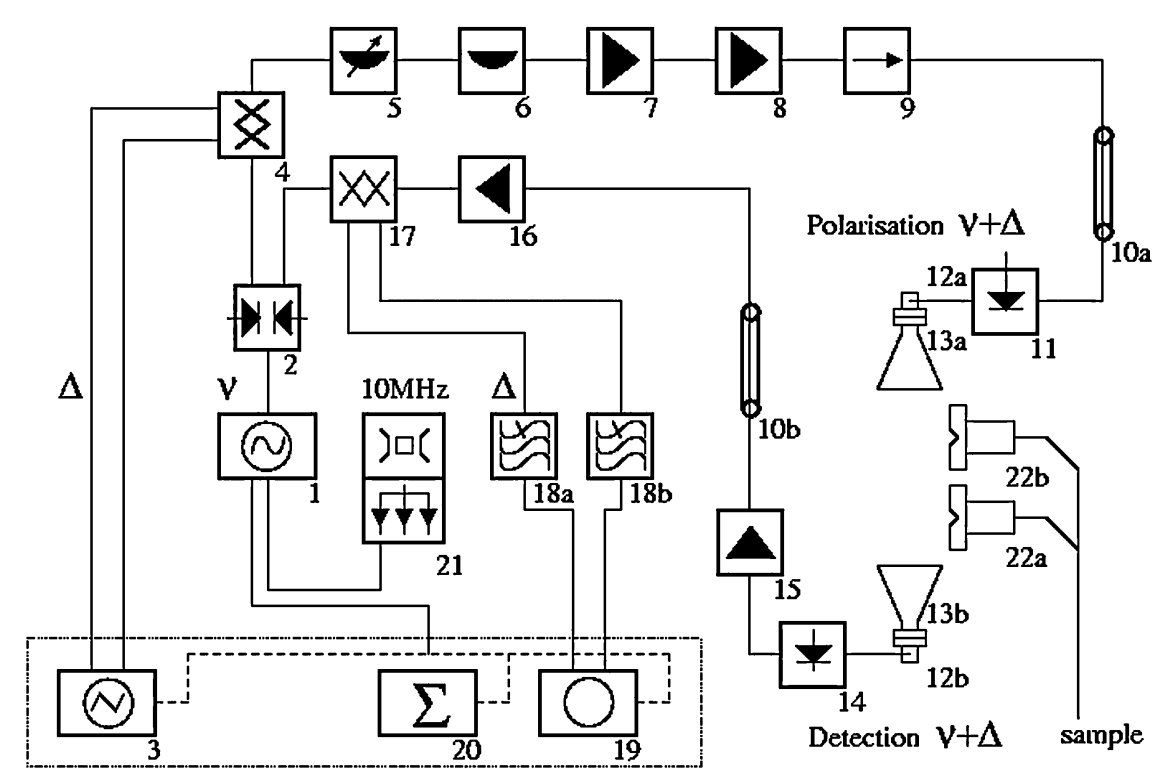

Scheme 1 Supersonic-jet expansion IMPACT spectrometer. (1) MW signal generator, HP8672A, 2-18 GHz, 10 dBm output power; (2) MW SPDT pin-diode switch, Sierra Microwave Technology SFD0526-001S, 0.5-26 GHz, $50 \mathrm{~dB}$ isolation, $3 \mathrm{~dB}$ insertion loss; (3) RF arbitrary-waveform generator, Agilent technologies N6030A, $2 \times 1.25 \mathrm{Gs} \mathrm{s}^{-1}$ generation, 15 bit resolution; (4) MW I/Q-upconverter, Miteq SM0226LC1MDQ, 2-26 GHz, $15 \mathrm{~dB}$ carrier suppression, $12 \mathrm{~dB}$ insertion loss; (5) MW variable attenuator NARDA 4798, 7-18 GHz, 0-20 dB; (6) MW attenuator, MiniCircuits, dc - $18 \mathrm{GHz}, 10 \mathrm{~dB}$; (7) MW amplifier, ALS0618-20-08, 6-18 GHz, $2.7 \mathrm{~dB}$ noise figure, $20 \mathrm{~dB}$ gain, $8 \mathrm{dBm}$ output power; (8) MW very-high-power TWT amplifier, Varian VZM6991K3, 8-18 GHz, $35 \mathrm{~dB}$ noise figure, $40 \mathrm{~dB}$ gain, $20 \mathrm{~W}$ output power; (9) MW isolator, El Dorado 18774, 8-18 GHz; (10) MW coaxial cable, HUBER-SUNHER SUCOFLEX, flexible; (11) MW SPST pin-diode Switch HP 33144A, 0.1-18 GHz, $80 \mathrm{~dB}$ isolation; (12) MW coaxial-waveguide adaptor, A-INFO 90WCAS, 8-12.4 GHz; (13) MW horn antenna, A-INFO JXTXLB-90-20, 8-12.4 GHz, 20 dBi gain; (14) MW power limiter, Agilent Technologies N9356C, 0.01-26.5 GHz, $25 \mathrm{dBm}$ threshold, $4 \mathrm{~W}$ input power; (15) MW very-low-noise amplifier, Miteq JSK4-06001800-35-10, 6-18 GHz, $1.5 \mathrm{~dB}$ noise figure, $35 \mathrm{~dB}$ gain, $10 \mathrm{dBm}$ output power; (16) MW amplifier, JCA0618-301, 6-18 GHz,3.9 dB noise figure, $15 \mathrm{~dB}$ gain, $10 \mathrm{dBm}$ output power; (17) MW I/Q-downconverter, Miteq IRM0226LC1Q, 2-26 GHz, $18 \mathrm{~dB}$ image rejection, $14.5 \mathrm{~dB}$ insertion loss; (18) RF lowpass filter, Reactel 10L3-495 S11, DC-495 MHz, 2 dB insertion loss; (19) RF Digitizer, LeCroy PXD1022, $2 \times 2 \mathrm{Gs} \mathrm{s}^{-1}$ real-time acquisition; (20) PXI-chassis based experiment control, data-acquisition, and -analysis system, National Instruments 1045/6602/6221/8195; (21) $10 \mathrm{MHz}$ frequency standard, Stanford Research FS725, $10 \mathrm{MHz}$ output frequency, $5 \times 10^{-10}$ frequency stability; (22) solenoid valves, General Valve series 9.

Since the extremely weak molecular signal is usually far below the thermal noise level, phase-synchronized repetitions are essential in order to achieve an improvement in the signalto-noise ratio by the phase-correct addition of individual measurements. A signal provided from a rubidium frequency standard (21) is used for the generation of all analog frequency signals and to derive all digital clock rates. Only if all signal sources used in the system are referenced to the same frequency standard, it becomes possible to generate the molecular signal with recurring phase. Since the relative arrangement of the molecular jet and the high-frequency field is perpendicular, the transit time of the polarized molecular jet is quite short and line widths of the order of $30 \mathrm{kHz}$ full-width-half-maximum (FWHM) are achieved. The signal frequency can be determined to be as accurate as $3 \mathrm{kHz}$ or $0.0000001 \mathrm{~cm}^{-1}$ for unblended lines. For nicotine, the molecular free-induction-decay (FID) was sampled at a rate of $1 \mathrm{GHz}$ for a period of $100 \mu$ s with $33 \mathrm{dBm}$ polarization power.

\section{(b) COBRA-FTMW}

The resonator-enhanced supersonic-jet FTMW spectrometer, described elsewhere, ${ }^{18}$ covering the frequency range of $4-18 \mathrm{GHz}$, was used to record the spectrum with the resolution necessary to analyze the hyperfine structure due to the presence of two ${ }^{14} \mathrm{~N}$ nuclei. The optimal conditions to polarize the molecules in the jet correspond to molecular pulses of about $1.1 \mathrm{~ms}$, followed by MW polarization pulses of $0.3 \mu$ duration with powers of 1-40 mW. The microwave transient FID was recorded for $100 \mu \mathrm{s}$ in the time domain at 40-100 ns sample intervals and Fourier-transformed to the frequency domain. Since the axes of the molecular beam and the resonator axes are co-linear in the coaxially oriented beam-resonator arrangement, each observed individual transition appears as a Doppler doublet. The frequency of each transition was determined by the arithmetic mean of the two Doppler components. The accuracy of frequency measurements was estimated to be better than $5 \mathrm{kHz}$.

\section{Results and discussion}

\section{(a) Model calculations}

The nicotine molecule $\left(\mathrm{C}_{10} \mathrm{H}_{14} \mathrm{~N}_{2}\right.$, see Fig. 1) is formed by a pyridine and a $N$-methyl pyrrolidine ring linked by a single bond. The possible conformations associated with the ring puckering motions of the pyrrolidine ring and the internal rotation of both parts of the molecule lead to a number of plausible conformations. Theoretical calculations predict the four stable conformers ${ }^{33}$ depicted in Table 1. To label these 


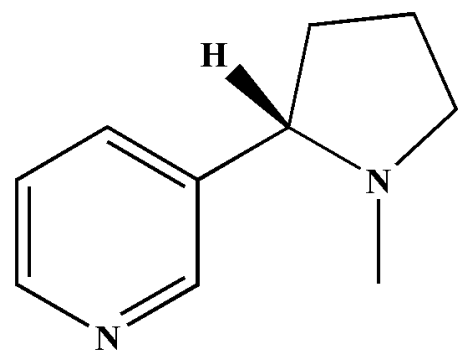

Fig. 1 Sketch of nicotine.

conformers we account for the cis or trans configurations of the methyl and pyrrolidine rings and the two possible configurations ( $a$ and $b$ ) obtained by rotation of pyridine with respect to the five membered ring.

To guide the spectral assignments, the results of quantumchemical calculations have been used to obtain the rotational constants, the quadrupole coupling constants and electric dipole moments in order to predict the frequencies, hyperfine patterns and intensities of nicotine rotational transitions. The geometries of these four low-energy structures were first reoptimized in a full energy calculation using second-order Møller-Plesset perturbation theory (MP2) ${ }^{34}$ in the frozen core approximation with Pople's 6-311++G(d,p) basis set. ${ }^{35}$ To differentiate conformers close in energy, a calculation including the zero-point vibrational contributions derived using the B3LYP functional in the harmonic approximation was performed, these values are reported in Table 1 along with calculated rotational constants, quadrupole coupling constants and dipole moment components. The predicted global minimum is the trans- $a$ conformer, followed in energy ordering by the trans- $b$ at $208 \mathrm{~cm}^{-1}$ using this method. Accordingly, the equilibrium mixture of nicotine should contain significant amounts of these two conformers at room temperature and they both should be present in the supersonic expansion of our experiment.

In addition, nicotine possesses two ${ }^{14} \mathrm{~N}$ nuclei with a nonzero quadrupole moment $(I=1)$ which interact with the electric field gradient at the site of the nuclei giving rise to a complicated hyperfine structure with many components. Because it reflects the orientation of the gradient with respect to the molecule's principle inertia axis system, each of the conformations adopted by nicotine would show a different hyperfine structure described by distinct values for the quadrupole coupling constants. If the resolution achieved in the experiment is high enough to fully resolve the quadrupole coupling hyperfine structure, we have an independent approach for a conclusive identification of the conformations present in the supersonic expansion.

\section{(b) Rotational spectra}

The conformers of nicotine are near prolate asymmetric tops with appreciable values of the $\mu_{\mathrm{a}}$ electric dipole moment component as reported in Table 1 . Their rotational spectra are predicted to exhibit an interesting trend: R-branch $\mu_{\mathrm{a}}$-type

Table 1 Predicted spectroscopic parameters for the low energy conformers of nicotine from ab initio MP2/6-311++G(d,p) calculations

\begin{tabular}{|c|c|c|c|c|}
\hline & trans- $a^{a}$ & trans- $b^{a}$ & $c i s-b$ & $c i s-a$ \\
\hline$A^{b} / \mathrm{MHz}$ & 2063 & 2049 & 2252 & 2257 \\
\hline$B / \mathrm{MHz}$ & 580 & 584 & 601 & 598 \\
\hline \multirow[t]{2}{*}{$\mathrm{C} / \mathrm{MHz}$} & 551 & 555 & 533 & 541 \\
\hline & $Q \mathrm{~N}_{\mathrm{m}}$ & $Q \mathrm{~N}_{\mathrm{m}}$ & $Q \mathrm{~N}_{\mathrm{m}}$ & $Q \mathrm{~N}_{\mathrm{m}}$ \\
\hline$\chi_{\mathrm{aa}}^{c} / \mathrm{MHz}$ & 2.18 & 2.10 & 2.05 & 1.91 \\
\hline$\chi_{\mathrm{bb}} / \mathrm{MHz}$ & 2.66 & 2.60 & -2.97 & -3.40 \\
\hline \multirow[t]{2}{*}{$\chi_{\mathrm{cc}} / \mathrm{MHz}$} & -4.84 & -4.70 & 0.93 & 1.49 \\
\hline & $Q \mathrm{~N}_{\mathrm{p}}$ & $Q \mathrm{~N}_{\mathrm{p}}$ & $Q \mathrm{~N}_{\mathrm{p}}$ & $Q \mathrm{~N}_{\mathrm{p}}$ \\
\hline$\chi_{\mathrm{aa}} / \mathrm{MHz}$ & -1.13 & 0.91 & 0.16 & -0.04 \\
\hline$\chi_{\mathrm{bb}} / \mathrm{MHz}$ & 3.14 & 3.18 & -2.45 & -2.65 \\
\hline$\chi_{\mathrm{cc}} / \mathrm{MHz}$ & -2.02 & -4.09 & 2.29 & 2.68 \\
\hline$\mu_{\mathrm{a}}^{d} / \mathrm{D}$ & 2.3 & -1.5 & -1.9 & -2.0 \\
\hline$\mu_{\mathrm{b}} / \mathrm{D}$ & -0.2 & 0.4 & 1.0 & -2.7 \\
\hline$\mu_{\mathrm{c}} / \mathrm{D}$ & -1.3 & -2.6 & 0.4 & -0.9 \\
\hline$\mu_{\text {total }} / \mathrm{D}$ & 2.6 & 3.1 & 2.2 & 3.5 \\
\hline$\Delta E_{\mathrm{MP}^{e}} / \mathrm{cm}^{-1}$ & 0 & 208 & 2009 & 2193 \\
\hline
\end{tabular}

${ }^{a}$ The experimentally observed conformers. For notation see the text. ${ }^{b} A, B$ and $C$ are the rotational constants. ${ }^{c}$ The ${ }^{14} \mathrm{~N}$ quadrupole coupling constants were obtained using the conversion: $\chi_{\alpha \alpha} / \mathrm{MHz}=2.34965\left(Q / \mathrm{fm}^{2}\right)\left(q_{\alpha \alpha} / \mathrm{au}\right)$, where $q_{\alpha \alpha}$ is the corresponding element of the electric field gradient at the $\mathrm{N}$ nucleus and the quadrupole coupling moment for ${ }^{14} \mathrm{~N}$ is taken to be $Q=2.09 \mathrm{fm}^{2}$, after P. L. Cummings, G. P. Bacskay, N. S. Hush, R. Aldrich, J. Chem. Phys., 1987, 86, 6908. Only diagonal elements are shown. $\mathrm{N}_{\mathrm{m}}$ corresponds to the nitrogen bonded to the methyl group and $\mathrm{N}_{\mathrm{p}}$ corresponds to the pyridinic nitrogen. ${ }^{d}$ Electric dipole moment.1 D $\approx 3.3356 \times 10^{-30} \mathrm{C} \mathrm{m} .{ }^{e}$ Relative energies (MP2/6-311++G(d,p) basis set) with respect to the global minimum. 
transitions occur in groups regularly spaced in frequency intervals nearly equal to $B+C$. The initial data sets were generated using the IMPACT spectrometer. The simultaneous observation of $1 \mathrm{GHz}$ regions of the spectrum greatly aided in the assignment. Hence, two sets of R-branch $\mu_{\mathrm{a}}$-type transitions were readily identified as corresponding to two different rotamers of nicotine labeled I and II. The spectra collected in the frequency range of $7500 \mathrm{MHz}$ to $9500 \mathrm{MHz}$ displayed in Fig. 2 correspond to values of $J=7 \leftarrow 6$ and $J=8 \leftarrow 7$ for the two observed rotamers. No lines attributable to other conformers remained unassigned in the spectrum at this level of sensitivity.
All transitions should exhibit a quadrupole hyperfine structure characteristic of the nuclear quadrupole coupling interaction originated by the presence of two ${ }^{14} \mathrm{~N}$ nuclei $(I=1)$. The nuclear spin of the two ${ }^{14} \mathrm{~N}$ nuclei couples to the rotational angular moment resulting in a complicated hyperfine structure with many components. We label the nitrogen of the pyrrole ring and that of the pyridinic ring as ${ }^{14} \mathrm{~N}_{\mathrm{m}}$ and ${ }^{14} \mathrm{~N}_{\mathrm{p}}$, respectively. As it is shown in Fig. 2, the resolution of the IMPACT spectrometer, due to the perpendicular arrangement between the supersonic jet and the resonator axis, is not sufficient to analyze this hyperfine structure. For this reason, only the central frequencies were measured and used in an iterative process to subsequently predict

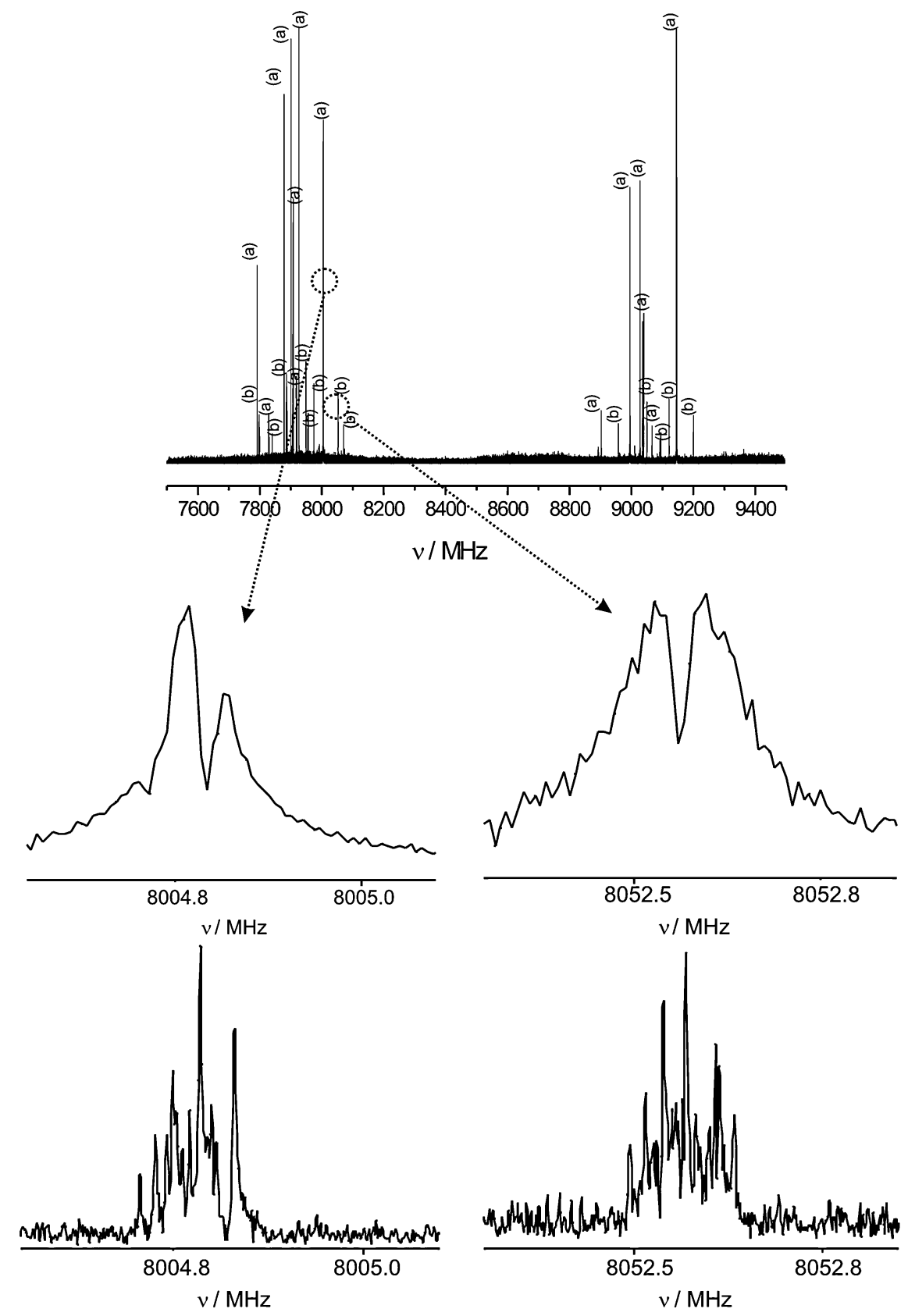

Fig. 2 Top: IMPACT rotational spectrum of nicotine, the lines labelled with (a) and (b) belong to conformers trans $a$ and trans $b$, respectively; center: detailed IMPACT spectra of $7_{1,7} \leftarrow 6_{1,6}$ transitions for conformers trans $a$ (right) and trans $b$ (left) of nicotine; bottom: COBRA spectra of $7_{1,7} \leftarrow 6_{1,6}$ transitions showing the quadrupole components labelled for conformers trans $a$ (right) and trans $b$ (left) of nicotine. 
and measure new lines. In addition, R-branch $\mu_{\mathrm{a}}$ - and $\mu_{\mathrm{c}}$-type transitions were predicted and measured for both rotamers leading to rotational constant sets of similar quality as those previously reported. ${ }^{23}$ A comparison of these experimental values with those in Table 1 leads to the conclusion that the two detected rotamers I and II are trans conformers of nicotine. The most intense spectrum was that of rotamer I and could correspond to the predicted lowest energy conformer, trans- $a$, which additionally has the larger value of $\mu_{\mathrm{a}}$. Consequently, rotamer II would correspond to conformer trans- $b$. The absence of $\mu_{\mathrm{b}}$-type spectra for both rotamers is consistent with the small values of the corresponding dipole moment component predicted for trans conformers (see Table 1).

Despite the spectroscopic analysis above, a definitive identification of a particular conformer cannot be made considering only the rotational constants. As seen in Table 1, their values are very close for the two conformers. Conformer trans- $a$ is structurally similar to conformer trans- $b$; they only differ by a rotation of $180^{\circ}$ about the bond between the two ring moieties. This only changes the relative position of the $\mathrm{N}_{\mathrm{p}}$ atom and does not alter substantially the mass distribution of the molecule. The nuclear quadrupole coupling interactions hyperfine structure depends strongly on the electronic environment of the nitrogen atom and its orientation with respect to the principal inertial axis system. One can see from Table 1 that the diagonal elements of the quadrupole coupling tensor $\chi_{\mathrm{aa}}$ and $\chi_{\mathrm{cc}}$ of the ${ }^{14} \mathrm{~N}_{\mathrm{p}}$ offer the best opportunity to discriminate between the trans conformers. Indeed, $\chi_{\mathrm{aa}}$ exhibits opposite signs for the two conformers and they would show a qualitatively very different hyperfine structure which can be precisely analyzed to determine the experimental values of the quadrupole coupling constants.

In the next step of the investigation, on the basis of the rotationally assigned spectrum, nicotine was probed by utilizing the high resolution of our COBRA-FTMW spectrometer. ${ }^{18}$ The frequency domain displays the same positional features (see Fig. 2 for $7_{1,6}-6_{1,5}$ rotational transition) but the spectral widths of the resonances in the COBRA-FTMW spectrum are noticeably smaller due to the coaxial arrangement of the supersonic jet with respect to the resonator. ${ }^{17}$ The hyperfine structure was then resolved for all observed rotational transitions. Up to 182 and 129 hyperfine components were measured for I and II rotamers, respectively. The transition frequencies are given in Tables S1 and S2 of ESI. $\dagger$ They were fitted ${ }^{36}$ using a Watson's S-reduced semirigid rotor Hamiltonian $H_{\mathrm{R}}{ }^{(\mathrm{S})}$ in the $I^{r}$ representation, ${ }^{37}$ supplemented with a $H_{Q}$ term to account for the nuclear quadrupole coupling contribution. ${ }^{38}$ The Hamiltonian was set up in the coupled basis set $\left(I_{1} I_{2} I J F\right), I_{1}+I_{2}=I, I+J=F$ and diagonalized in blocks of $F .^{39}$ The energy levels involved in each transition are thus labeled with the quantum numbers $J, K_{-1}, K_{+1}, I, F$. The experimentally determined rotational constants $A, B$, and $C$ and the quadrupole coupling constants $\chi_{\mathrm{aa}}, \chi_{\mathrm{bb}}$, and $\chi_{\mathrm{cc}}$ for both rotamers are given in Table 2 . It is now possible to distinguish unambiguously among the trans- $a$ and trans- $b$ conformers of nicotine. The nuclear quadrupole coupling constants of the ${ }^{14} \mathrm{~N}_{\mathrm{p}}$ nucleus of the pyridinic ring provide conclusive assignments. Hence, rotamers I and II are unambiguously identified as trans- $a$ and trans- $b$ conformers
Table 2 Spectroscopic parameters for the observed conformers of nicotine

\begin{tabular}{lll}
\hline & trans- $a$ & trans- $b$ \\
\hline$A^{a} / \mathrm{MHz}$ & $2059.56318(65)^{d}$ & $2045.68314(49)$ \\
$B / \mathrm{MHz}$ & $579.87099(12)$ & $583.257764(87)$ \\
$C / \mathrm{MHz}$ & $549.32304(11)$ & $552.824597(97)$ \\
$D_{\mathrm{J}} / \mathrm{kHz}$ & $25.49(78)$ & $25.92(75)$ \\
$D_{\mathrm{JK}} / \mathrm{kHz}$ & $223.0(53)$ & $220.8(87)$ \\
\hline & $Q \mathrm{~N}_{\mathrm{m}}$ & $Q \mathrm{~N}_{\mathrm{m}}$ \\
\hline & $2.1311(91)$ & $2.0431(98)$ \\
$\chi_{\mathrm{aa}} / \mathrm{MHz}$ & $2.572(38)$ & $2.574(19)$ \\
$\chi_{\mathrm{bb}} / \mathrm{MHz}$ & $-4.703(38)$ & $-4.617(19)$ \\
$\chi_{\mathrm{cc}} / \mathrm{MHz}$ & $Q \mathrm{~N}_{\mathrm{p}}$ & $Q \mathrm{~N}_{\mathrm{p}}$ \\
\hline & $-1.1509(95)$ & $0.8559(97)$ \\
\hline$\chi_{\mathrm{aa}} / \mathrm{MHz}$ & $3.3292(56)$ & $3.188(18)$ \\
$\chi_{\mathrm{bb}} / \mathrm{MHz}$ & $-2.1784(56)$ & $-4.044(18)$ \\
$\chi_{\mathrm{cc}} / \mathrm{MHz}$ & 3.5 & 3.1 \\
$\sigma^{b} / \mathrm{kHz}$ & 182 & 129 \\
$\mathrm{~N}^{c}$ & &
\end{tabular}

${ }^{a} A, B$ and $C$ are the rotational constants; $D_{\mathrm{J}}$ and $D_{\mathrm{JK}}$ are the quartic centrifugal distortion constants; $\chi_{\mathrm{aa}}, \chi_{\mathrm{bb}}$, and $\chi_{\mathrm{cc}}$ are the diagonal elements of the ${ }^{14} \mathrm{~N}$ nuclear quadrupole coupling tensor. $\mathrm{N}_{\mathrm{m}}$ corresponds to the nitrogen bonded to the methyl group and $\mathrm{N}_{\mathrm{p}}$ corresponds to the pyridinic nitrogen. ${ }^{b}$ rms deviation of the fit. ${ }^{c}$ Number of fitted hyperfine components. ${ }^{d}$ Standard error in parentheses in the units of the last digit.

respectively. Given the good agreement between observed and calculated spectroscopy constants, the ab initio structures can be taken as a good description of the actual structure of the conformers of nicotine. These structures are consistent with the results of the earlier ED study. ${ }^{13}$

Apart from those due to the Doppler effect, no additional splitting due to the internal rotation was observed in the spectra. To rationalize this, we again made use of $a b$ initio calculations to predict the energy barriers for the internal rotation of the methyl groups. These have been obtained by performing single point energy calculations for incremental values of the dihedral angle between the methyl rotor and the rest of the molecule. The methyl group barriers were predicted to be 1362 and $1364 \mathrm{~cm}^{-1}$ for the trans- $a$ and trans- $b$ conformers, respectively. Given these values, the predicted splittings in the spectrum are narrower than the $7 \mathrm{kHz}$ resolution attained in our COBRA-FTMW spectrometer, which support their absence in the spectrum.

Trans configurations similar to those of nicotine have been detected for the related compounds 2-phenylpyrrolidine ${ }^{14}$ and anabasine. ${ }^{15}$ In all cases the rotation of the aromatic ring around the $\mathrm{C}-\mathrm{C}$ bond gives rise to a double minimum potential function. In the case of 2-phenylpyrrolidine, due to the symmetry of the phenyl group, both minima are equivalent and as a consequence only one conformer is detected. In nicotine and anabasine the pyridine ring is bonded to the pyrrolidine or piperidine through the meta carbon and this gives rise to two non-equivalent minima and thus to two different conformers.

\section{Conclusions}

We have observed and identified two conformers of nicotine in a neon supersonic expansion. Conclusive identification of the 
conformers was achieved by comparing the accurate experimental values of the rotational and nuclear quadrupole coupling constants with those predicted in vacuo by ab initio calculations. Nuclear quadrupole coupling constants of the nitrogen atom $\left(\mathrm{N}_{\mathrm{p}}\right)$ of the side pyridine ring have been used to establish the position of this nitrogen atom within the molecule. This is of great relevance, because the orientation of the nitrogen site then allows discrimination between the two trans conformers with similar rotational constants. As has been already shown in previous studies, ${ }^{40}$ the determination of quadrupole coupling constants constitutes a unique tool in the identification of conformers or tautomers of biomolecules when rotational constants are not conclusive enough. The two conformers present $N$-methyl trans configurations with the two rings approximately perpendicular to each another.

Broadband (chirp) and narrowband (pulse) FTMW spectroscopies have provided complementary information leading to a definitive structural assignment of the two conformers. Despite the advantages of the new IMPACT instrument in rapidly collecting spectra and facilitating assignments, its resolution is not yet sufficient to fully resolve quadrupole hyperfine components of nitrogen in nicotine. Future work will address this in an attempt to reduce the line width because nitrogen is frequently encountered in species of bio- or physiological importance. By employing parabolic reflectors, a quasi-coaxial arrangement of the molecular jet and the high-frequency electromagnetic field similar to that of the COBRA-FTMW apparatus can be reached. With this configuration, the components of the Doppler doublet are expected to have a linewidth on the order of that of the COBRA-FTMW experiments.

\section{Acknowledgements}

This research was funded by the Ministerio de Ciencia $e$ Innovación (Grants CTQ 2006-05981/BQU, CTQ2010-17436, and Consolider-Ingenio 2010 CSD2009-00038), Junta de Castilla y León (Grant VA070A08). I.P. and C.C. thank the Ministerio de Ciencia e Innovación for FPI Grants. We thank the authors of ref. 23 for making available the values of rotational constants of nicotine that they determined through the abstract collection of the International Symposium on Molecular Spectroscopy, Columbus, Ohio, USA.

\section{References}

1 P. Dome, J. Lazary, M. P. Kalapos and Z. Rihmer, Neurosci. Biobehav. Rev., 2010, 34, 295, and references therein.

2 M. Tomizawa and J. E. Casida, Acc. Chem. Res., 2009, 42, 260.

3 W. Lewis and P. J. Steel, Supramol. Chem., 2005, 17, 579.

4 C. Chothia and P. Pauling, Proc. Natl. Acad. Sci. U. S. A., 1970, 65, 477.

5 T. P. Pitner, W. B. Edwards, R. L. Bassfield and J. F. Whidby, J. Am. Chem. Soc., 1978, 100, 246.

6 J. I. Seeman, Heterocycles, 1984, 22, 165.

7 J. F. Whidby, W. B. Edwards and T. P. Pitner, J. Org. Chem., $1979,44,794$.

8 R. H. Cox, J. Kao, H. V. Secor and J. I. Seeman, J. Mol. Struct., 1986, 140, 93 .

9 R. J. Radna, L. Beveridg and A. L. Bender, J. Am. Chem. Soc., 1973, 95, 3831 .
10 J. Graton, M. Berthelot, J. F. Gal, S. Girard, C. Laurence, J. Lebreton, J. Y. Le Questel, P. C. Maria and P. Naus, J. Am. Chem. Soc., 2002, 124, 10552.

11 M. Seydou, G. Gregoire, J. Liquier, J. Lemaire, J. P. Schermann and C. Desfrancois, J. Am. Chem. Soc., 2008, 130, 4187.

12 C. Mihesan, M. Ziskind, C. Focsa, M. Seydou, F. Lecomte and J. P. Schermann, Int. J. Mass Spectrom., 2008, 277, 284.

13 T. Takeshima, R. Fukumoto, T. Egawa and S. Konaka, J. Phys. Chem. A, 2002, 106, 8734.

14 D. E. Martin, E. G. Robertson, J. G. MacLellan, P. D. Godfrey, C. D. Thompson and R. J. S. Morrison, J. Am. Chem. Soc., 2009, 131, 2638.

15 A. Lesarri, E. J. Cocinero, L. Evangelisti, R. D. Suenram, W. Caminati and J.-U. Grabow, Chem.-Eur. J., 2010, 16, 10214.

16 T. J. Balle and W. H. Flygare, Rev. Sci. Instrum., 1981, 52, 33.

17 J.-U. Grabow, W. Stahl and H. Dreizler, Rev. Sci. Instrum., 1996, 67, 4072 .

18 J. L. Alonso, F. L. Lorenzo, J. C. López, A. Lesarri, S. Mata and H. Dreizler, Chem. Phys., 1997, 218, 267.

19 (a) S. Antolínez, A. Lesarri, S. Mata, S. Blanco, J. C. López and J. L. Alonso, J. Mol. Struct., 2002, 612, 125; (b) A. Lesarri, S. Mata, S. Blanco, J. C. López and J. L. Alonso, J. Chem. Phys., 2004, 120, 6191; (c) A. Lesarri, S. Mata, J. C. López and J. L. Alonso, Rev. Sci. Instrum., 2003, 74, 4799.

20 J. L Alonso, C. Pérez, M. E. Sanz, J. C. López and S. Blanco, Phys. Chem. Chem. Phys., 2009, 11, 617, and references therein.

21 S. Blanco, J. C. López, J. L. Alonso, P. Ottaviani and W. Caminati, J. Chem. Phys., 2003, 119, 880.

22 J. L. Alonso, M. E. Sanz, J. C. López and V. Cortijo, J. Am. Chem. Soc., 2009, 131, 4320.

23 R. J. Lavrich, R. D. Suenram, F. Plusquellic and S. Davis, 58th International Symposium on Molecular Spectroscopy, Columbus, Ohio, USA, 2003, comm. RH13.

24 J.-U. Grabow, S. Mata, J. C. López, I. Peńa, C. Cabezas, S. Blanco, J. L. Alonso, 65th International Symposium on Molecular Spectroscopy, Columbus, Ohio, USA, 2010, comm. WH06.

25 G. G. Brown, B. C. Dian, K. O. Douglass, S. M. Geyer and B. H. Pate, J. Mol. Spectrosc., 2006, 238, 200.

26 G. G. Brown, B. C. Dian, K. O. Douglass, S. M. Geyer, S. T. Shipman and B. H. Pate, Rev. Sci. Instrum., 2008, 79, 053103 .

27 G. S. Grubbs, C. T. Dewberry, K. C. Etchison, K. E. Kerr and S. A. Cooke, Rev. Sci. Instrum., 2007, 78, 096106.

28 J.-U. Grabow, Applikationsschrift, GWLU Hannover, available on-line at: http://www.ft-mw.org/.

29 J.-U. Grabow, Fourier Transform Microwave Spectroscopy: Measurement \& Instrumentation, in Handbook of High-Resolution Spectroscopies, ed. Martin Quack and Frédéric Merkt, John Wiley \& Sons, Chichester, 2011, pp. 723-799.

30 U. Andresen, H. Dreizler, J.-U. Grabow and W. Stahl, Rev. Sci. Instrum., 1990, 61, 3694.

31 J.-U. Grabow, E. S. Palmer, M. C. McCarthy and P. Thaddeus, Rev. Sci. Instrum., 2005, 76, 093106.

32 J.-U. Grabow and W. Stahl, Z. Naturforsch., A: Phys. Sci., 1990, 45, 1043

33 D. E. Elmore and D. A. Dougherty, J. Org. Chem., 2000, 65, 742.

34 C. Moller and M. S. Plesset, Phys. Rev., 1934, 46, 618.

35 M. J. Frisch, et al., Gaussian03 (Revision B.04), Gaussian, Inc., Pittsburgh, PA, 2003.

36 H. M. Pickett, J. Mol. Spectrosc., 1991, 148, 371.

37 J. K. G. Watson, in Vibrational Spectra and Structure, ed. J. R. Durig, Elsevier, New York, Amsterdam, 1977, vol. 6, pp. 1-78.

38 W. Gordy and R. L. Cook, Microwave Molecular Spectra, John Wiley \& Sons, New York, 3rd edn, 1984.

39 (a) H. M. Foley, Phys. Rev., 1947, 71, 747; (b) G. W. Robinson and C. D. Cornwell, J. Chem. Phys., 1953, 21, 1436.

40 (a) M. E. Sanz, A. Lesarri, M. I. Peña, V. Vaquero, V. Cortijo, J. C. López and J. L. Alonso, J. Am. Chem. Soc., 2006, 128, 3812; (b) V. Vaquero, M. E. Sanz, J. C. López and J. L. Alonso, J. Phys. Chem. A, 2007, 111, 3443; (c) J. C. López, M. I. Peña, M. E. Sanz and J. L. Alonso, J. Chem. Phys., 2007, 126, 191103. 